\title{
Velocity-Based Stability Margins for Fast Bipedal Walking
}

\author{
Jerry E. Pratt ${ }^{1}$ and Russ Tedrake ${ }^{2}$ \\ 1 Florida Institute for Human and Machine Cognition jpratt@ihmc.us \\ 2 Massachusettes Institute of Technology russt@mit.edu
}

\section{Abstract}

We present velocity-based stability margins for fast bipedal walking that are sufficient conditions for stability, allow comparison between different walking algorithms, are measurable and computable, and are meaningful. While not completely necessary conditions, they are tighter necessary conditions than several previously proposed stability margins. The stability margins we present take into consideration a biped's Center of Mass position and velocity, the reachable region of its swing leg, the time required to swing its swing leg, and the amount of internal angular momentum available for capturing balance. They predict the opportunity for the biped to place its swing leg in such a way that it can continue walking without falling down. We present methods for estimating these stability margins by using simple models of walking such as an inverted pendulum model and the Linear Inverted Pendulum model. We show that by considering the Center of Mass location with respect to the Center of Pressure on the foot, these estimates are easily computable. Finally, we show through simulation experiments on a 12 degree-of-freedom distributed-mass lower-body biped that these estimates are useful for analyzing and controlling bipedal walking.

\section{Introduction}

"How stable is your robot?" is a fundamental yet challenging question to answer, particularly with fast moving legged robots, such as dynamically balanced bipedal walkers. With many traditional control systems, questions of stability and robustness can be answered by eigenvalues, phase margins, loop gain margins, and other stability margins. However, legged robots are nonlinear, under-actuated, combine continuous and discrete dynamics, and do not necessarily have periodic motions. These features make applying traditional stability margins difficult. 
In this paper we define stability for a biped simply as whether or not the biped will fall down. We focus on velocity-based stability since we believe that regulating the velocity of the Center of Mass is the most challenging subtask for human-like bipedal walking. Regulating velocity is a challenging subtask due to the extended period during a natural gait that the Center of Mass velocity is underactuated (the actuators cannot produce an arbitrary acceleration on the Center of Mass). For example, once the body has traveled far enough away from the foot, the only course of action that can stabilize the Center of Mass velocity is to take a step. Other requirements such as regulating virtual leg length and body orientation, and swinging the swing leg, can be met through traditional control system techniques since these subtasks are fully actuated during the majority of the gait.

We argue that the most crucial thing for regulating velocity in bipedal walking is the ability to place the foot of the swing leg in a proper location that allows for maintaining or reducing velocity on subsequent steps. A sufficient condition for being able to maintain or reduce velocity is the ability to eventually come to a stop. Thus we define stability margins that estimate the likelihood that a biped can "Capture" its kinetic energy and stop over a given number of steps. We define a Capture Point as a point that can be stepped to in order to stop. We propose the "N-Step Capture Stability Margin" which gives an indication of the degree to which a biped can stop in $\mathrm{N}$ steps. For most practical purposes if a walking biped cannot stop within several steps, it is probably close enough to falling to consider it unstable. Hence being, say, 10-Step Capturable is a sufficient, and close to necessary, stability condition.

Exact computation of these stability margins is difficult since the dynamics of bipedal walking is complex. However, we can compute these margins for simplified walking models. These simplified walking models give useful approximations to the real values of the margins, which we demonstrate through control experiments on a simulated 12 degree-of-freedom lower-body biped with distributed mass. The robot can recover from being pushed by stepping to a Capture Point computed from the simplified walking models. It can step to desired foothold locations by guiding the Capture Point to the desired stepping point and stepping once the Capture Point reaches it.

\section{Stability Definition}

In this paper, we define stability for a biped in terms of whether or not the biped will fall down. However, the concept of falling down is difficult to precisely define. For example, sitting down on the floor and slipping down onto the floor might result in the exact same trajectories and end state but one is considered falling and the other is considered sitting, with the only difference being intent.

For the purposes of this paper, to eliminate complications rising from such concepts as intent, let us define a fall as follows. 
Definition 1 (Fall). When a point on the biped, other than a point on the feet of the biped, touches the ground.

This definition allows for such things as holding a hand railing to not be considered a fall, by pushing the burden onto a precise definition of the ground and of the feet. However, in this paper, we only consider situations in which the ground is everything that is not part of the biped and the feet are the two commonly known appendages at the end of the legs.

Turning now to the question of whether the biped will fall, consider the deterministic closed-loop dynamics of the biped in the general form $\dot{\mathbf{x}}=f(\mathbf{x}, t)$, where $\mathbf{x} \in \Re^{N}$ is the state vector. Define a subset $F \subset \Re^{N}$ which includes all configurations of the robot for which some part of the robot other than the feet is touching the ground. The basin of attraction of $F$, which we will call the "Basin of Fall", defines all of the states of the robot that eventually lead to a fall:

Definition 2 (Basin of Fall). Subset of state space that leads to a fall. $B \subset$ $\Re^{N}, x(t) \in B \Rightarrow \exists \Delta t \geq 0$ s.t. $\mathbf{x}(t+\Delta t) \in F$

The complement of the Basin of Fall is the Viability Kernel of Wieber [32]. Whether or not the robot will fall down, and hence whether or not the robot is stable, can now be precisely defined.

Definition 3 (Stable). A biped is stable if and only if the state of the robot is not inside the Basin of Fall, B.

Note that for a real-world biped in a non-deterministic environment, the Basin of Fall may be the entire state-space, as all bipeds will eventually fall given enough time. Also, note that for a biped that has regions of chaotic gait, the Basin of Fall may be Uncomputable as determining whether some states are in the Basin of Fall may be Undecideable [26]. In addition, for the system to be deterministic, the dynamics, $f$, and the state vector, $\mathbf{x}$, must contain full information about the environment such as the ground profile. Encoding the entire environment for all time is prohibitive in general.

Therefore, to ensure computability, non-zero volume of stable states, and feasible definition of the state and environment, one may wish to consider the state of the biped to be stable if it does not lead to a fall after some reasonable finite amount of time, $\tau$, and define the Time-Limited Basin of Fall as follows:

Definition 4 (Time-Limited Basin of Fall). Subset of state space that leads to a fall within a finite amount of time, $\tau . B_{\tau} \subset \Re^{N}, x(t) \in B_{\tau} \Rightarrow$ $\exists \Delta t, 0 \leq \Delta t \leq \tau$ s.t. $\mathbf{x}(t+\Delta t) \in F$

For human-like walking, approximately 1 minute is a reasonable horizon to consider since it is highly unlikely that a biped would be in a state where a fall is inevitable but the biped can stagger around before the fall for a whole minute. 
Computing the Basin of Fall, $B$, is conceptually simple, but computationally expensive. Given an accurate closed-loop dynamic model and a discretized state space, one can use dynamic programming to determine $B$. Let $V(\mathbf{x})$ be the value function encoding the time until the biped falls. Initialize $V$ to zero for states that are falling states and infinity for all other states. Then use the update rule $V\left(\mathbf{x}_{\mathbf{n}}\right)=\min \left(V\left(\mathbf{x}_{\mathbf{n}}\right), V\left(\mathbf{x}_{\mathbf{n}+\mathbf{1}}\right)+\Delta t\right)$ where state $x_{n}$ dynamically leads to state $x_{n+1}$ and $\Delta t$ is the time taken to transition from state $x_{n}$ to $x_{n+1}$. In the worst case $N^{2}$ updates would have to occur, where $N$ is the number of discrete states. If the dynamics can be simulated backward in time, then only $N$ updates need be made if the states are visited recursively, starting from falling states. In a companion paper [28], we will use similar ideas to explore stochastic stability margins for legged locomotion.

A stability margin that logically follows from the previous discussion is the distance to the Basin of Fall, called the Viability Margin by Wieber [32].

$$
M_{\text {Viability }}(\mathbf{x})=\left\{\begin{array}{r}
\min _{\mathbf{x}^{\prime} \in B}\left(\left|\mathbf{x}-\mathbf{x}^{\prime}\right|\right) \text { if } \mathbf{x} \notin B \\
-\min _{\mathbf{x}^{\prime} \notin B}\left(\left|\mathbf{x}-\mathbf{x}^{\prime}\right|\right) \text { if } \mathbf{x} \in B
\end{array}\right.
$$

By definition the Viability Margin is both a necessary and sufficient indicator of stability and thus would be a good stability margin for a biped. However, it does have drawbacks. It does not take into consideration what disturbances we would like the biped to be robust to, since distance in state space does not necessarily correlate to real-world disturbances. It does not take into consideration the dynamics of the system in moving from the current state to the nearest boundary state, since Cartesian nearness does not necessarily correlate with dynamic nearness. It assigns importance to each degree of freedom based upon its units of measure. It is difficult to compute. And finally, it does not give us much insight into why a biped is stable or why it falls. Some of these problems could be mitigated by such things as weighting the state variables based on importance, for each state computing the magnitude of a given disturbance required to transition the biped into a Basin of Fall state, etc. However, these additions would only add to the computational complexity of computing this margin.

Additionally, perfect dynamic models are impossible to attain for real systems, and to compute the entire Basin of Fall during experimental trials would require an infeasible number of trials, many of which could result in damage to the biped.

Due to these limitations, instead of attempting to compute the entire Basin of Fall, in this paper we endeavor to develop heuristic stability margins that approximate whether a state is in the Basin of Fall. These margins, such as the N-Step Capture Margin, give an indication of the ability of the biped to come to a stop within a given number of steps. Previous stability margins, such as the static stability margin, are similar heuristic approximations, but are typically too conservative. In the next section we discuss desirable characteristics for heuristic bipedal walking stability margins. 


\section{Desirable Characteristics of Stability Margins}

An ideal stability margin for a biped would act as a fortune teller. It would tell us when the biped is going to fall down next, what the cause will be, and how it can be prevented. If the biped is not going to fall down, the margin would indicate the closest the biped will be to falling down in the next step or so, at what point during the gait this occurs, and how much extra disturbance it could handle.

While such omniscience is infeasible for anything but the simplest systems, some reasonable characteristics we may desire for stability margins include:

- Necessary. If the stability margin is outside an acceptable threshold of values, the robot will fall down.

- Sufficient. If the stability margin is inside the acceptable threshold of values, the robot will not fall down.

- Comparable. Two control algorithms should be comparable for stability based on their relative stability margins.

- Measurable and Computable. One should be able to measure the relevant state variables and estimate the stability margin on-line in order to use it for control purposes.

- Meaningful. The stability margin should answer relevant questions as to why the robot fell. It should correlate with the degree of robustness to disturbances, such as noise, terrain irregularities, and external forces or impulses.

The Viability Margin is necessary, sufficient, and allows comparisons. However, its main drawback is that it is very difficult to compute. Various heuristic stability margins, which are much easier to compute, have been used in analyzing and controlling bipeds. In the next section we review some of the margins that are commonly used for bipedal walking and discuss how well they achieve these desirable characteristics. In the subsequent sections we introduce some heuristic stability margins which we believe more accurately measure the stability of a biped as defined by Definition 3 .

\section{Review of Stability Margins for Bipedal Walking}

While there have been many proposed ways to define stability for a bipedal walking robot, we argue that many of these do not adequately address the desired characteristics described above. Here we review eigenvalues of Poincare return maps [14], the Zero Moment Point (ZMP) criterion [30], the foot rotation indicator (FRI) [7], and change of angular momentum [1, 20] as potential stability margins in light of the desired characteristics. 


\subsection{Eigenvalues of Poincare Return Maps}

For a periodic system, trajectories in state space will cycle and return each cycle to a point in a slice of state space. Deviations from a periodic limit cycle will return to the cycle, and for small deviations, typically follow a linear relation,

$$
X_{n+1}=K X_{n}
$$

where $X$ is the vector of deviations from the fixed point that the limit cycle passes through and $K$ is a linear return matrix. One of the eigenvalues of $K$, corresponding to the evolution of the orbit, will be 1.0. If the magnitude of the remaining eigenvalues of $K$ are all less than one, then the limit cycle is stable. Thus, the magnitude of the largest eigenvalue of the return map, disregarding the eigenvalue corresponding to the orbit, is a suitable stability margin for a periodic system. Measuring the eigenvalues of Poincare return maps is commonly used for analyzing Passive Dynamic Walking robots [16, $8,27,2,4,29]$ and was used by Miura and Shimoyama [17] to analyze their Biper robots.

However, using eigenvalues of Poincare return maps assumes periodicity and is valid only for small deviations from a limit cycle. While most bipedal systems, including humans, indeed seem to be periodic, there is nothing about the bipedal walking problem that requires periodicity. In particular, a biped's motion is not periodic when walking over discontinuous rough terrain, or when abruptly changing speed or direction of travel. In addition, large disruptions from a limit cycle, such as when being pushed, cannot be analyzed using this technique as it assumes small deviations. Therefore, while eigenvalue magnitudes of Poincare return maps may be sufficient for analyzing periodic bipedal walking, they are not sufficient for analyzing nonperiodic motions, and are not necessary for analyzing bipedal walking in general.

\subsection{Zero Moment Point (ZMP) and Foot Rotation Indicator (FRI)}

The Zero Moment Point (ZMP) is the location on the ground where the net moment generated from the ground reaction forces has zero moment about two axes that lie in the plane of the ground [30]. The ZMP when used in control algorithm synthesis for bipedal walking robots typically is computed analytically based upon desired trajectories of the robot's joints. As long as the ZMP lies strictly inside the support polygon of the foot, then these desired trajectories are dynamically feasible. If the ZMP lies on the edge of the support polygon, then the trajectories may not be dynamically feasible.

During playback of the desired joint trajectories, the actual ZMP is measured from force sensors in the foot or by observing accelerations of all the joints [10]. Then deviations between the precomputed and actual ZMP are typically used to modify the joint trajectories $[34,9]$. The ZMP is equivalent 
to the Center of Pressure $(\mathrm{CoP})$ but is commonly used to mean the analytically computed point based on the state and acceleration of the robot whereas the CoP is commonly used to mean the point measured from ground reaction forces.

The Foot Rotation Indicator (FRI) point [7] is the point on the ground where the net ground reaction force would have to act to keep the foot stationary given the state of the biped and the accelerations of its joints. If the foot is stationary, then the FRI, the ZMP, and the CoP are all the same point. If the foot is experiencing rotational acceleration, then the ZMP and CoP are on an edge of the support polygon, and the FRI is outside the support polygon. Therefore the FRI is a more general form of the ZMP and provides both a positive and negative margin when used for control and analysis purposes.

The ZMP stability margin is the distance from the ZMP to the nearest edge of the convex hull of the support polygon. In its typical use it measures how much room for error there is in achieving the desired trajectories of the robot. If the ZMP reaches the edge of the support polygon, then the desired Center of Mass trajectory may no longer be dynamically feasible.

Maintaining the ZMP inside the support polygon is not a necessary condition for stable walking. During toe-off in human walking and in walking of the robot Spring Flamingo [23], the ZMP stability criterion is violated, the FRI point lies outside the foot, and the foot rotates. For bipeds with point feet [11, 24, 5, 31], and Passive Dynamic Walkers with curved feet, when on one support foot, the ZMP, FRI, and CoP have little value as they are all simply the location of the foot, and the ZMP criterion is always violated. Maintaining the ZMP inside the support polygon is also not a sufficient condition for stable walking since a biped can fall down while its ZMP remains in the center of its foot. As an example, if all the joint torques are set to zero, the robot will collapse while the ZMP stays inside the foot.

The ZMP criterion does become a sufficient criterion for stable walking when coupled with a particular class of control algorithms, typically those that rely on stiff tracking of predetermined joint trajectories [13, 34, 9, 19]. One way to state the ZMP criterion is: Given desired state variable trajectories that are consistent with the dynamics and that predict the ZMP staying inside the support polygon, a trajectory tracking control algorithm can stably track those trajectories as long as the ZMP does indeed remain inside the support polygon.

In these terms the ZMP criterion is a sufficient criterion for stable walking and has become a very powerful tool for trajectory generation and verification of the dynamic feasibility of trajectories during execution. However, its utility has perhaps led to its overuse, resulting in the majority of bipedal robots relying heavily on prerecorded trajectories and stiff joint control to achieve those trajectories. Such stiff joint control of prerecorded trajectories typically leads to poor robustness to pushes and unknown rough terrain, relies on a flatfooted gait, and makes it difficult to incorporate natural dynamic mechanisms 
that have shown their utility in Passive Dynamic Walkers [16, 8, 27, 2, 4, 29], and a growing number of powered bipeds [22, 3, 33].

Since the ZMP criterion is a sufficient condition for stable walking only when coupled with a particular class of control system, as pointed out by Abdallah and Goswami [1] the ZMP criterion should be viewed as a controllability criterion that assesses the dynamic feasibility of a desired trajectory through state-space.

It is important to note that the ZMP, CoP, and FRI are not functions of the state variables of the robot, but rather require knowledge of either the joint torques and/or the joint accelerations. Thus, they themselves cannot be viewed as state variables, and thus are not good candidates for stability margins that are a function of the state of the robot. Instead, for this paper and in previous work [23, 22], we view the Center of Pressure as a control input that can be arbitrarily and instantaneously changed, within the limits of actuator bandwidth, force limits, and the limitation that it stays inside the support polygon.

\subsection{Angular Momentum}

Motivated by observations that humans appear to regulate angular momentum about the Center of Mass when standing, walking, and running, researchers $[1,20]$ have suggested that angular momentum about the Center of Mass (refered to as spin angular momentum) of a biped should be minimized throughout a motion. They argue that the spin angular momentum should be used when needed to balance, such as when lunging to prevent a fall after being pushed, or windmilling one's arms when standing on a balance beam. The amount of angular momentum that is available is limited by joint angle limits, joint speed limits, and joint power limits. By minimizing angular momentum when not required, the biped has a reserve it can draw on when necessary.

Minimizing spin angular momentum is not a necessary condition for stable walking. One can walk while violently thrashing his or her upper body mass around. It is not graceful, nor efficient, and perhaps grace and efficiency are the primary reasons humans do not usually walk in this fashion. Minimizing spin angular momentum is also not a sufficient condition for stable walking, as a biped can fall over while maintaining an angular momentum of zero. Therefore, we believe angular momentum about the Center of Mass, taken purely on its own, is not a good indicator of whether a biped will fall and hence not a good stability margin by itself.

However, the reserve in spin angular momentum that can be utilized to help recover from a push or other disturbance is important, as there is a coupling between angular momentum rate change and linear momentum rate change, and hence speed. This coupling arises from the fact that the net angular momentum rate change of the biped, about the Center of Pressure, is only modified by gravity (Figure 1). Since the ground reaction force, by 


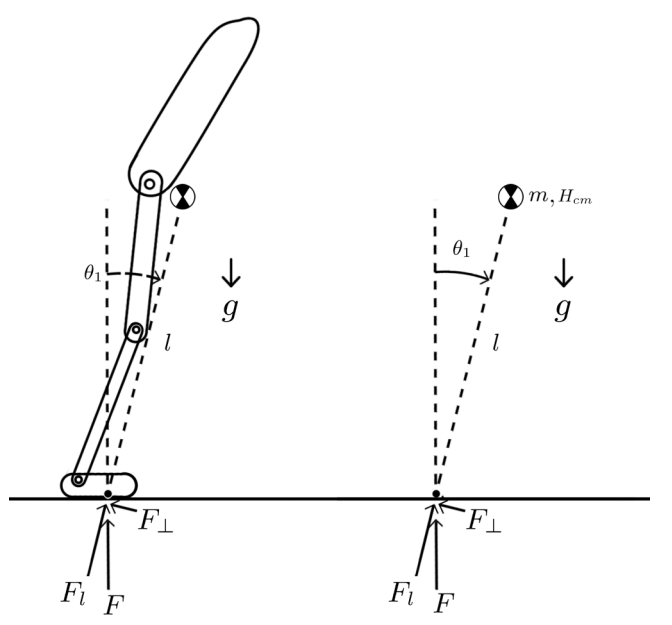

Fig. 1. Bipedal model for sagittal plane dynamics. $l$ is the virtual leg length from the Center of Pressure to the Center of Mass. $\theta_{1}$ is the angle from vertical to the virtual leg. $F$ is the ground reaction force, with component $F_{l}$ along the virtual leg and $F_{\perp}$ perpendicular to it. $m$ and $H_{c m}$ are the mass and angular momentum about the Center of Mass of the biped. $g$ is the gravitational acceleration constant.

definition, acts through the Center of Pressure it does not affect the angular momentum about this point. The angular momentum dynamics about the Center of Pressure can therefore be written as

$$
\dot{H}_{t o t}=m g l \sin \theta_{1},
$$

where $H_{\text {tot }}$ is the total angular momentum about the Center of Pressure, $m$ is the mass of the robot, $g$ is the gravitational acceleration, $l$ is the distance from the Center of Pressure to the Center of Mass, and $\theta_{1}$ is the angle from the Center of Pressure to the Center of Mass with vertical being zero. The total momentum about the Center of Pressure consists of the angular momentum of the Center of Mass rotating about the Center of Pressure, plus the spin angular momentum about the Center of Mass:

$$
H_{t o t}=H_{0}+H_{c m}=m l^{2} \dot{\theta}_{1}+H_{c m} .
$$

Differentiating, we get

$$
\dot{H}_{t o t}=m g l \sin \theta_{1},=m l^{2} \ddot{\theta}_{1}+2 m l i \dot{\theta}_{1}+\dot{H}_{c m} .
$$

The first term, $m l^{2} \ddot{\theta}_{1}$ is the acceleration of the Center of Mass penduluming around the Center of Pressure. The second term, 2 mli $\dot{\theta}_{1}$ encodes the coupling of distance to Center of Mass and rotational velocity (which makes a person rotate faster when they pull their legs in on a tire swing). Since 
only gravity has an influence on $\dot{H}_{t o t}$, equation 5 demonstrates the coupling between acceleration of spin angular momentum about the Center of Mass, $\dot{H}_{c m}$, and acceleration of the Center of Mass about the Center of Pressure, $\ddot{\theta}_{1}$. A clockwise acceleration of internal inertia will create a counterclockwise acceleration of the Center of Mass penduluming over the Center of Pressure and vice versa. That is what leads to the counterintuitive strategy of lunging your upper body forward to prevent falling forward.

Since spin angular momentum is useful for catching balance in this way, having a reserve is useful. However, instead of using the reserve of spin angular momentum as a stability margin by itself, in Section 8 we propose using it as it relates to reducing the velocity of the biped. In the next section we justify our focus on velocity for analyzing bipedal stability.

\section{Bipedal Walking and Stability}

There are five subtasks that a biped performs while walking.

T1 Maintain body orientation within a reasonable bound.

T2 Maintain virtual leg length within a reasonable bound.

T3 Swing the swing leg.

T4 Transfer support from one support leg to the other.

T5 Regulate Center of Mass velocity.

For human-like bipedal walking, subtasks T1 through T3 are achievable through traditional control means as long as the support leg is firmly planted and joint torque limits that prevent foot slippage are observed. While there are occasions during the gait cycle where these tasks are not fully controllable, such as perhaps at the end of toe-off, we argue that there is plenty of opportunity during a normal bipedal walking gait to control orientation, virtual leg length, and leg swing using traditional techniques that depend on full controllability and full observability.

Subtask T4, transfer of support, is difficult when attempted using traditional high-gain joint position control techniques due to the over-constrained kinematics that the resultant closed loop kinematic structure presents [25]. However, when low impedance force control techniques are used [23], the system is no longer over-constrained and smooth transfer of support can occur relatively easily.

We contend that the most difficult subtask in bipedal walking from a controllability point of view is subtask T5, regulating the Center of Mass velocity. It is this subtask that makes bipedal walking both an interesting and challenging problem. It is the most difficult subtask as the degrees of freedom that contribute to the velocity vector are under-actuated from a continuous dynamics point of view. Once the Center of Mass projection on the ground moves even a small distance out of the support polygon of the feet, then a significant amount of angular acceleration of internal mass, such as windmilling 
the arms or lunging the upper body, must occur to bring the Center of Mass back. Beyond a certain distance, the Center of Mass cannot be brought back at all and the biped must take a step. Thus velocity can be regulated only through a combination of the continuous dynamics and the discrete dynamics. It is this lack of actuation and the requirement of taking a step to continue walking that leads many to describe walking as a sequence of controlled falling.

We can illustrate the difficulty of regulating velocity versus orientation and leg length by looking at the dynamics of walking during single support. To simplify the discussion, we consider only sagittal plane dynamics (Figure 1 ), but our discussion extends to 3D dynamics. The dynamics of the rotation of the mass about the Center of Pressure (Equation 5) is

$$
\dot{H}_{t o t}=m g l \sin \theta_{1}=m l^{2} \ddot{\theta}_{1}+2 m l i \dot{\theta}_{1}+\dot{H}_{c m}
$$

where $l$ is the virtual leg length from the Center of Pressure to the Center of Mass, $\theta_{1}$ is the angle from vertical to the virtual leg, $m$ and $H_{c m}$ are the mass and angular momentum about the Center of Mass of the biped, and $g$ is the gravitational acceleration constant.

As discussed in Section 5, humans tend to regulate their angular momentum about the Center of Mass, and thus the magnitude of $\dot{H}_{c m}$ is kept relatively low. Suppose $\dot{H}_{c m}=0$. Since the virtual leg length, $l$ is always positive, then equation 6 can be rewritten as

$$
\ddot{\theta}_{1}=-c_{1} i \dot{\theta}_{1}+c_{2} \sin \theta_{1}
$$

where $c_{1}$ and $c_{2}$ are always positive. If $\dot{\theta}_{1}$ and $\theta_{1}$ have the same sign, then the magnitude of $\theta_{1}$ must always be increasing, assuming $|i|<\infty$ and $-\pi<\theta_{1}<$ $\pi$. In physical terms, this means that if the Center of Mass is moving away from the Center of Pressure, it cannot be stopped without either moving the location of the Center of Pressure (instantaneoulsy changing the value of $\theta_{1}$ ), or by accelerating internal inertia. However, once the Center of Mass is beyond the support polygon of the foot, changing the Center of Pressure alone cannot stop further motion of the Center of Mass. At that point, only accelerating internal inertia $\left(\dot{H}_{c m} \neq 0\right)$, or taking a step can prevent a fall. Due to joint range of motion, velocity, and torque limits, the amount of opportunity for using angular momentum to catch balance is limited. Therefore, during a large portion of a human-like walking gait, the only way to prevent a fall is to take another step.

Turning to the dynamics of the virtual leg length from the Center of Pressure to the Center of Mass we have

$$
m \ddot{l}=m l \dot{\theta}_{1}^{2}-m g \cos \theta_{1}+F_{l}
$$

where $F_{l}$ is the component of the ground reaction force along the line from the Center of Pressure to the Center of Mass and is typically positive since the total ground reaction force must be positive and lie inside the friction cone. $F_{l}$ 
is a function of the leg actuator forces and as long as the leg isn't straight can be arbitrarily and instantaneously set to any positive value, assuming ideal force-source actuators, as long as it doesn't result in slipping on the ground. Since $m g$ is typically larger than $m \dot{l}_{1}{ }^{2}$ on the Earth at typical walking speeds, one can achieve a large range of negative $\ddot{l}$, and an even larger range of positive $\ddot{l}$, bounded only by the strength of the leg. Therefore, during a large percentage of the walking gait, the virtual leg length, $l$ is fully controllable.

Turning to the dynamics of the angular momentum about the Center of Mass we have

$$
\dot{H}_{c m}=l F_{\perp}
$$

where $F_{\perp}$ is the component of the ground reaction force perpendicular to the line from the Center of Pressure to the Center of Mass. $F_{\perp}$ is a function of the leg actuator forces and can be arbitrarily and instantaneously set to any value, assuming ideal force-source actuators, as long as it doesn't result in slipping on the ground. Since a large range of positive and negative values of $\dot{H}_{c m}$ can be achieved during a large portion of the walking gait, angular momentum about the Center of Mass is fully controllable.

Note that in this discussion we do not break out the components of $H_{c m}$ due to their complexity, nor do we consider the motion of internal degrees of freedom. We assume that all the internal degrees of freedom have an actuator associated with them, and only consider their net effect on the walking motion as it couples through $H_{c m}$. Achieving any orientation of internal body parts, or swinging the swing leg along a trajectory in internal coordinates is thus achievable with traditional control means.

Note that in this discussion we use $\dot{\theta}_{1}$ as our velocity variable (and could use $\dot{\theta}_{2}$ for a $3 \mathrm{D}$ analysis). However, any two variables that are independent of virtual leg length velocity, $i$ could be used. Except for a fall, the virtual leg should never lie in the horizontal plane. Therefore, $\dot{x}$ and $\dot{y}$ would also be a suitable choice in the above analysis. As long as we choose a definition of a velocity vector that along with the virtual leg vector spans the $3 \mathrm{D}$ space, the above analysis can be modified to show that velocity is not fully controllable for a large percentage of a human-like walking gait.

We have shown that virtual leg length and body orientation are fully controllable during a large portion of a natural gait, with the main limitation in their control being the requirement that the foot doesn't slip on the ground. In contrast, velocity is not controllable with continuous dynamics once the Center of Mass has moved away from the support polygon. For human-like walking, velocity is controllable only through a combination of continuous dynamics and discrete dynamics (i.e. taking a step). This lack of controllability is why we consider velocity regulation the most challenging part of walking and why we focus on stability margins were velocity is central. 


\section{Capture Points and Capture Regions}

A key capability required for robust 3D walking is the ability to place the swing leg foot at an appropriate position, such that the Center of Mass can come to rest over the foot. We call such a point a "Capture Point". Before defining a Capture Point, we define a Capture State and a Safe Feasible Trajectory:

Definition 5 (Capture State). State in which the kinetic energy of the biped is zero and can remain zero with suitable joint torques.

Definition 6 (Safe Feasible Trajectory). Trajectory through state space that is consistent with the robot's dynamics, is achievable by the robot's actuators, and does not contain any Falling States.

Note that the Center of Mass must lie above the support polygon in a Capture State. We now define a Capture Point:

Definition 7 (Capture Point). For a biped in state $x$, a Capture Point, $p$, is a point on the ground where if the biped covers $p$, either with its stance foot or by stepping to $p$ in a single step, and then maintains its Center of Pressure to lie on $p$, then there exists a Safe Feasible Trajectory that ends in a Capture State.

Note that since height, swing, orientation, and velocity are dynamically coupled, the location of a Capture Point is dependent on the trajectory through state-space before and after swinging the leg and thus is not a unique point. Therefore, there exists a Capture Region on the ground such that if the Center of Pressure is placed inside this region, then the biped can come to a stop for some height and orientation trajectory.

Definition 8 (Capture Region). The set of all Capture Points.

The size of the Capture Region is highly dependent on how fast the swing leg can swing to a Capture Point before the biped has accelerated and the point is no longer a Capture Point. The faster the swing leg, the larger the area of the Capture Region. Therefore, all else being equal, a biped with a faster swing leg will have a higher margin of stability than one with a slower swing leg.

If constraints are placed on the subsequent height, swing, orientation, and internal motion of the robot, then the size of the Capture Region will be reduced. For example, we can define a momentum-regulated capture region as the set of all Capture Points that exist when $\dot{H}_{c m}$ is regulated during the subsequent motion. With enough constraints, the Capture Region may be reduced to a single point, or perhaps even vanish.

Note that our definition of Capture Point also implies that the swing leg can reach the point. Let us define an Unreachable Capture Point as a point that is not a Capture Point, but would be if the swing leg did not have 
kinematic constraints. The Unreachable Capture Region is then the set of all Unreachable Capture Points and there is no intersection between the Unreachable and Reachable Capture Regions.

While being able to stop in one step implies stability by definition 3 , it is overconservative. Being able to stop in two steps is a less conservative estimate of stability and hence we consider Two-Step Capture Points.

Definition 9 (Two-Step Capture Point). A point on the ground, $p$, such that if the biped swung its swing leg to cover $p$ with its foot and maintained its Center of Pressure to lie on p, then there exists a Safe Feasible Trajectory, such that at some state along the trajectory, there exists a Capture Point.

Definition 10 (Two-Step Capture Region). The set of all Two-Step Capture Points.

We can now define an N-Step Capture Point recursively:

Definition 11 (N-Step Capture Point). A point on the ground, $p$, such that if the biped swung its swing leg to cover $p$ with its foot and maintained its Center of Pressure to lie on p, then there exists a Safe Feasible Trajectory, such that at some state along the trajectory, there exists an N-1-Step Capture Point.

Definition 12 (N-Step Capture Region). The set of all N-Step Capture Points.

If an N-Step Capture point exists, then we say that the biped is "N-Step Capturable". As N approaches $\infty$, the N-step Capture Region converges to the area on the ground that the foot can be placed at without resulting in an eventual fall.

Note that the above definitions require the maintenance of the Center of Pressure at a Capture Point, which is not necessary nor typical in walking. More general definitions would allow the Center of Pressure to move inside the foot. However, we conjecture that such definitions would result in equivalent Capture Regions, i.e. if a biped can stop by taking a step and then moving its Center of Pressure around its foot, then the biped can stop by taking the same step and maintaining the Center of Pressure at a single point inside the foot. We chose to maintain the Center of Pressure at a Capture Point in our definitions for computational reasons since doing so reduces the potential action space by two degrees-of-freedom. However, in controlling a biped, there is no such requirement.

The stability margins we propose in the next Section will give a measure of how much opportunity there is to stop based on the ability to reach a Capture Region with the swing leg. Knowing where the leg can swing to will be important and so we define the Reachable Region as follows.

Definition 13. Reachable Region: Region on the ground that can be reached kinematically by any point on the bottom of the swing foot. 


\section{Proposed Stability Margins}

We now propose some velocity-based stability margins for bipedal walking that are based on Capture Regions. Because the Capture and Reachable Regions depend on the current state of the robot, $\mathbf{x}$, these margins are scalar functions of $\mathbf{x}$.

- Zero-Step Capture Margin. $M(\mathbf{x}, 0)$ : Maximum distance from points in the Capture Region to their closest edge of the support polygon if the Capture Region and support polygon intersect. Otherwise, the negative distance from the Capture Region to the support polygon.

- One-Step Capture Margin. $M(\mathrm{x}, 1)$ : Maximum distance from points in the Capture Region to their nearest boundary of the Reachable Region if the Capture Region is non-empty. Otherwise, the negative distance from the Unreachable Capture Region to the Reachable Region.

- N-Step Capture Margin. $M(\mathbf{x}, N)$ : Maximum distance from points in the N-Step Capture Region to their nearest boundary of the Reachable Region if the N-Step Capture Region is non-empty. Otherwise, the negative distance from the Unreachable N-Step Capture Region to the Reachable Region.

Note that the Zero-Step Capture Margin is equivalent to the traditional static stability margin when velocity is negligible and angular momentum is not used for capturing balance. For a moving biped, it is more appropriate than the traditional margin, since the Center of Pressure needs to be placed near the Capture Point rather than the ground projection of the Center of Mass to stop the biped.

The above margins assume that any dynamically feasible trajectory through the state space is acceptable. Variants of the above margins can be defined that impose constraints on the trajectories, control system, etc. For example, angular-momentum-regulated versions of these margins such as the AngularMomentum-Regulated N-Step Capture Margin, $M\left(\mathbf{x}, N,\left|\dot{H}_{c m}\right|<\dot{H}_{c m_{\max }}\right)$, can be defined as above, but where the Capture Region is computed with the assumption that angular momentum is limited for capturing balance. These margins are useful for determining whether the robot is Capturable without having to rely on drastic measures such as lunging or windmilling.

All of the above margins are in terms of how close the biped is to being able to stop. Determining if a biped is able to stop over a number of steps is appropriate, since if a biped cannot slow down and eventually stop, then its state is likely in the Basin of Fall, except for some metastable border states on the boundary of the Basin of Fall. We conjecture that in the limit as $\mathrm{N}$ approaches infinity, N-Step Capturable implies stability as defined by Definition 3. For most practical purposes, if the biped cannot stop in several steps, it is probably close enough to falling to consider it unstable. Therefore, though still not a completely necessary criterion, being say 10-step Capturable should be a suitable stability criterion. 
The above stability margins are all in terms of Cartesian distance of various regions on the ground to the Reachable Region. This distance is related to the ability to get the swing leg to the region and also the time before the region moves and is no longer reachable if it isn't stepped to rapidly. One could also define other margins in terms of the area of a given region. These margin would give an indication of the degree of accuracy required in placing the swing leg in the region. For example,

- N-Step Area-Based Capture Margin. $M_{\text {area }}(\mathbf{x}, N)$ : Area of the NStep Capture Region if the N-Step Capture Region is non-empty. Otherwise, the negative distance from the Unreachable N-Step Capture Region to the Reachable Region.

We have now suggested a number of potential stability margins that can be used for analyzing and controlling bipedal walking. In terms of the desirable characteristics listed in Section 4, the margins listed above are sufficient conditions for stability, allow comparisons between different walking algorithms, and are meaningful. While none of them, except for boundary cases requiring extreme computationally complexity, are tight necessary conditions, they are tighter necessary conditions than previously proposed stability margins. These margins are measurable and computable. For $N=1$, estimates with low computational complexity are easy to derive, as described in the next section. As $N$ increases, the computational requirements likely increase, but may be reasonable for small $N$.

Deciding which margin is the most desirable and what degree of stability is required depends on tradeoffs between performance and safety. For example, if the biped is walking carefully over stepping stones, then having a large One-Step Area-Based Capture Margin may be desirable. If the biped is a gymnast on a balance beam, then being Angular-Momentum-Regulated OneStep Capturable is important since lunging or windmilling to prevent a fall results in deductions. And if the biped is an Olympic Race Walker, being able to stop in a small number of steps is less important than speed, so a small degree of $\infty$-Step Capture Margin is all that may be required.

In the next section we will discuss methods for estimating Capture Point locations and some of these stability margins.

\section{Estimating Stability Margins}

In this section we describe some methods for estimating our proposed stability margins.

\subsection{Estimating One-Step Capture Points}

We can derive estimates of the location of Capture Points by using inverted pendulum models that approximate walking. Using a constant length inverted 
pendulum model (Figure 2, left side) and equating initial and final energy, we have $\frac{1}{2} m v^{2}+m g h_{0}=m g l$, where $m$ is the mass, $v$ is the velocity of the mass, $g$ is the gravitational constant, $h_{0}$ is the initial height above the ground, and $l$ is the virtual leg length. Let $\hat{s}$ be the unit vector parallel to the ground that points in the same direction as the Center of Mass velocity. The Capture Point will lie somewhere on the line defined by the projection of the Center of Mass on the ground and $\hat{s}: \mathbf{x}_{c}=r_{c} \hat{s}$, where $\mathbf{x}_{c}$ is the location of the Capture Point with respect to the ground projection of the Center of Mass and $r_{c}$ is the distance from the Center of Mass projection to the Capture Point. Because $r_{c}, l$, and $h_{0}$ form a right triangle, we have $r_{c}=\sqrt{l^{2}-h_{0}^{2}}$. Solving for $r_{c}$ we get

$$
r_{c}=v \sqrt{\left(\frac{h_{0}}{g}+\frac{v^{2}}{4 g^{2}}\right)}
$$
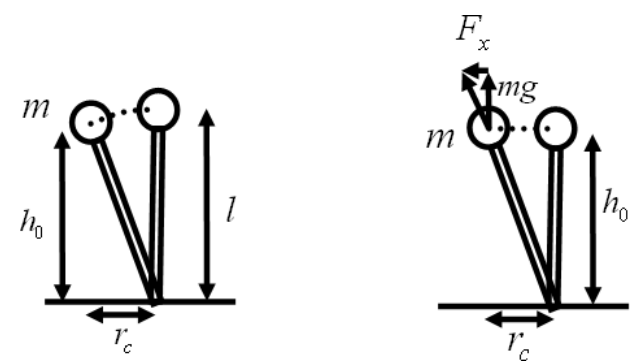

Fig. 2. Simple models for estimating the location of Capture Points.

This model assumes that the leg length stays constant as the Center of Mass follows an arc, coming to rest above the Capture Point. Another model, referred to as the Linear Inverted Pendulum model [11, 12], assumes that the Center of Mass height stays constant (Figure 2 - right side). Using this method to compute the location of a Capture Point results in an even simpler equation. In order to counter gravity, the vertical force on the mass must be $m g$. Because ground reaction forces can only act on the line between the Center of Pressure and the Center of Mass, similar triangles are formed and we have

$$
\frac{F_{x}}{m g}=\frac{x}{h_{0}} \Longrightarrow F_{x}=\frac{m g}{h_{0}} x
$$

where $F_{x}$ is the horizontal force on the mass, and $x$ is the distance from the mass to the Capture Point. Because the mass moves at a constant height, the energy absorbed while moving above the Capture Point will be the integral of the force times the displacement: 


$$
E=\int_{0}^{r_{c}} F d x=\frac{m g}{h_{0}} \int_{0}^{r_{c}} x d x=\frac{m g}{2 h_{0}} r_{c}^{2}
$$

Equating initial and final energies, we have $\frac{1}{2} m v^{2}=\frac{m g}{2 h_{0}} r_{c}^{2}$. Solving for $r_{c}$ we get

$$
r_{c}=v \sqrt{\frac{h_{0}}{g}}
$$

Equations 10 and 13 give estimates for two different Capture Points, one based on the Center of Mass following an arc, and one based on it following a straight horizontal line. Because the Center of Mass typically follows a flattened arc in bipedal walking, a more relevant Capture Point for a typical gait will likely fall between those two bounds. For parameter values typical in human walking, these two bounds are within $15 \%$ of each other.

The above estimates assumed that the swing leg could instantaneously arrive at the Capture Point. Since it may take some time for the swing leg to get to the Capture Point, we should estimate where the Capture Point will be when the swing leg arrives. If we have an estimate on the time remaining for swing, we can estimate the predicted Capture Point location using the Linear Inverted Pendulum model. Figure 3 illustrates the key variables in estimating the predicted Capture Point location. First we estimate the Center of Mass trajectory. From the previous analysis, $F_{x}=\frac{m g}{h_{0}} x$. Therefore, $\ddot{x}=\frac{g}{h_{0}} x$. Since this is a linear equation, it can be solved in closed form $[11,12]$

$$
x=C_{0} e^{w t}+C_{1} e^{-w t}, \dot{x}=D_{0} e^{w t}+D_{1} e^{-w t}
$$

where $w=\sqrt{\frac{g}{h_{0}}}$ and

$C_{0}=\frac{1}{2}\left(x_{0}+\frac{v_{0}}{w}\right), C_{1}=\frac{1}{2}\left(x_{0}-\frac{v_{0}}{w}\right), D_{0}=\frac{1}{2}\left(w x_{0}+v_{0}\right), D_{1}=\frac{1}{2}\left(-w x_{0}+v_{0}\right)$

The equations for $y$ are identical, with the proper substitutions. Given the estimated swing time, we can estimate the location and velocity of the Center of Mass at the end of swing using Equation 15. Using Equation 13 we then can estimate the location of the Capture Point at the end of swing. As the leg is swinging, we can update the predicted Capture Point location and adjust the swing leg trajectory to land in the desired location.

The above estimates of Capture Points were for point mass models. We can estimate a Capture Region using the above equations to compute the center of the region and then models for using angular momentum to determine a region around the center. Suppose we have no limit in how fast we can change our angular momentum $\left(\dot{H}_{c m}\right.$ is unbounded), but we do have a limit on the angular momentum about the Center of Mass that we have in "reserve", $H_{\text {reserve }}$ Also suppose we have a limit on the duration, $\tau_{\text {reserve }}$ that the biped can achieve that angular momentum before running into joint limits and having 


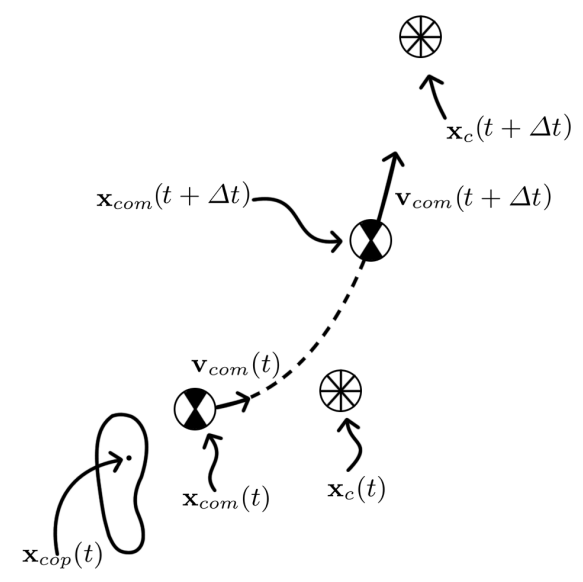

Fig. 3. Evolution of the Center of Mass and a Capture Point from time $t$ to $t+\Delta t$. $\mathbf{x}_{\text {cop }}(t)$ is the location of the Center of Pressure; $\mathbf{x}_{\text {com }}(t)$ and $\mathbf{v}_{\text {com }}(t)$ are the location and velocity of the Center of Mass; and $\mathbf{x}_{c}(t)$ is the location of the Capture Point.

to "pay it back" by stopping the rotation. We conjecture that once it has been determined to use reserve angular momentum to catch balance, immediately using all that is available as rapidly as possible is the strategy that results in the recovery from the worst conditions.

If we assume that the change in angular momentum is achieved instantaneously through an impulsive torque, then the change in rotational velocity of the Center of Mass about the Center of Pressure can be determined by integrating Equation 6 for an infinitesimal amount of time. The result is

$$
\Delta \dot{\theta}_{1}=-\frac{1}{m l^{2}} H_{\text {reserve }}
$$

Given the new velocity, we can estimate the Center of Mass trajectory during the next $\tau_{\text {reserve }}$ seconds using Equation 14. At the end of this time, the angular momentum must be "paid back" before joint angle limits are violated. We can then repeat the above steps to estimate the final Center of Mass position and velocity.

To estimate the Capture Region, one can use a search, guessing points on the boundary and checking with the above procedure to see if the Center of Mass can be captured over that point with the reserves of angular momentum. After several points on the boundary are determined, then the Capture Region can be estimated with a best-fit curve. 


\subsection{Estimating N-Step Capture Points}

Estimating N-Step Capture Points can be achieved through a brute-force search algorithm. Even with a large state space, the search can be relatively fast if $\mathrm{N}$ is small and a discrete step-to-step transition function, $S$ is known,

$$
\mathbf{x}_{n+1}=S\left(\mathbf{x}_{n}, \mathbf{x}_{\text {step }}, \mathbf{P}\right)
$$

where $\mathbf{x}_{n}$ is the state of the step $\mathrm{n}, \mathbf{x}_{\text {step }}$ is the location of the point that the biped steps to, and $\mathbf{P}$ are parameters that govern the motion of the step. The Linear Inverted Pendulum analysis described previously provides one such example of a discrete step-to-step transition function, with the time of step being the only input parameter.

Since it is likely that, on flat ground, an N-Step Capture Region is a connected, and perhaps even convex area on the ground, a search for the boundary of the Capture Region can be performed when considering the first $\mathbf{x}_{\text {step }}$. After the first step, then steps should only be considered that are likely optimal for stopping as quickly as possible. If a One-Step Capture Point exists, then the step should be there. If not, then the step should be as quickly as possible as far as possible toward the Unreachable Capture Region.

\subsection{Quick Estimates on the Number of Steps Required for a Stop}

We can estimate the approximate number of steps required for a biped to stop by estimating how much the biped can slow down each step using the Linear Inverted Pendulum model. The amount of energy absorbed during deceleration is $\frac{m g}{2 h_{0}} r_{\text {step }}$ and the amount of energy returned during acceleration is $\frac{m g}{2 h_{0}} r_{\text {accel }}$ where $r_{\text {step }}$ is the step length from Center of Mass projection on the ground to the Center of Pressure, and $r_{a c c e l}$ is the distance from the trailing support leg's Center of Pressure to the Center of Mass projection on the ground. If swing can happen quickly enough, $r_{a c c e l}$ can be zero. However, when the swing leg takes long enough to swing that exchange of support cannot be achieved before the Center of Mass passes over the trailing Center of Pressure, then $r_{\text {accel }} \neq 0 . r_{\text {accel }}$ can be estimated with Equation 14, given the required swing time. With the net change in energy we can then determine the velocity on the next step,

$$
v_{n+1}^{2}=v_{n}^{2}-\frac{g}{h_{0}} r_{\text {step }}^{2}+\frac{g}{h_{0}} r_{\text {accel }}^{2}
$$

We can iterate on Equation 18 to estimate the number of steps required to stop the biped. Note that a biped will always be able to slow down if the swing leg is fast enough to have a non-symmetric stance in which the Center of Pressure is in front of the Center of Mass more than it is behind it $\left(r_{\text {step }}>r_{\text {accel }}\right)$. When the swing leg takes too long to swing $\left(r_{\text {step }}<r_{\text {accel }}\right)$ the biped necessarily continues to speed up and eventually falls down. 
If $r_{\text {accel }}=0$, then the number of steps required to stop is $N=\frac{h_{0}}{g r_{\text {step }}^{2}} v^{2}$. If $r_{\text {accel }} \neq 0$ then $N>\frac{h_{0}}{g r_{\text {step }}^{2}} v^{2}$. For typical human parameters at fast walking speeds, $\frac{h_{0}}{g r_{\text {step }}^{2}} v^{2} \approx 1$ meaning a human at top speed usually requires 1 or 2 steps to come to a stop but typically not more. This estimate is easily verified through simple experiments in which one person walks fast while another calls out "stop" at a random time.

Note that a biped with its mass concentrated in its body, and no limit to the torques at its joint, should be able to walk extremely fast along a straight line trajectory. The only limit is how quickly it can swing its leg and transfer support. But with massless legs, it should be able to swing and transfer instantaneously. Along a flattened arc trajectory, the speed would be limited by the requirement of the centripetal acceleration of the Center of Mass being less than the acceleration of gravity. At higher velocities, the ground reaction force falls below zero and the biped becomes airborne. For humans walking at Earth's gravity, this limitation is not the limiting factor in top speed [22]. However, at the gravity of the moon, this limitation is relevant and explains why astronauts preferred to hop on the moon rather than walk.

\section{Standing Balance Strategies}

Using our proposed stability definitions we can now propose an algorithm for selecting an appropriate strategy to regain balance after being disturbed when standing. The algorithm below is for the reflex phase of balance recovery [1]. Once the robots velocity is captured, than a recovery phase can begin to restore the posture of the robot.

1. if Momentum-Regulated Zero-Step Capturable, then use the Center of Pressure to maintain balance.

2. else if Zero-Step Capturable, then use angular momentum to capture balance by lunging or windmilling.

3. else if Momentum-Regulated One-Step Capturable, then take a step to a Momentum-Regulated Capture Point and use the Center of Pressure to maintain balance after the step.

4. else if One-Step Capturable, then take a step to a Capture Point and use angular momentum to capture balance after the step.

5. else if N-Step Capturable, take a sequence of steps to capture balance.

6. else take a running step and/or fall.

The first two strategies have been well documented with human walking and are often refered to as the "ankle strategy" and the "hip strategy" [15]. We prefer to call them the "Center of Pressure strategy" and the "Angular Momentum Strategy". All of the strategies are easily observable in easily performable balance experiments. Using the stability margins proposed in 
this paper, it should be possible to hypothesize what amount of disturbance is required to cause a human to switch from one strategy to another one.

\section{Control Algorithms}

\subsection{Control Algorithms for Push Recovery}

The balance strategies listed above can be easily implemented in a control algorithm for push recovery. For such an algorithm, it is not important to accurately compute the various stability margins. What is more important is to rapidly determine which balance recovery method to attempt. If a step is required, reaction speed is critical and therefore, determining a good place to step is more appropriate than deliberating on the optimal place to step.

In a simulation study, we developed an algorithm that attempts to stay balanced on one foot. The simulated robot uses modulation of the Center of Pressure in order to keep the Capture Point, which is estimated every control cycle using the Linear Inverted Pendulum model, inside the foot. The commanded Center of Pressure location, $\mathbf{x}_{C o P}$ is computed using a linear controller on the desired Capture Point location, $\mathbf{x}_{\text {capture }}^{\prime}$,

$$
\mathbf{x}_{\text {CoP }}=\mathbf{x}_{\text {capture }}+k\left(\mathbf{x}_{\text {capture }}^{\prime}-\mathbf{x}_{\text {capture }}\right)
$$

where $k$ is the controller gain parameter. If the computed Center of Pressure location falls outside the foot, then it is changed to the nearest point on the foot's edge that lies on the line from the desired Capture Point to the actual Capture Point.

When a significant push occurs, the Capture Point moves outside of the foot. The biped approximates how long it will take to swing the leg to the point and uses that time in the estimate of where the Capture Point will be after swing. If this Capture Point is reachable, then the biped moves its Center of Pressure to the point on the foot nearest the Capture Point to minimize further acceleration and then takes a step to the Capture Point to regain balance. If this Capture Point is unreachable, then the algorithm assumes that if a 2-Step or N-Step Capture Region does exist, that it is near the boundary of the Reachable Region nearest the Unreachable Capture Point. Therefore, the biped will take a step as far as possible towards the Capture Point and then determine a new Capture Point to attempt to step to.

This algorithm works fairly well, validating the Capture Point estimates. When the robot is pushed in such a way that a Capture Point is reachable, the robot does regain balance on that step most of the time. This indicates that even if the estimated Capture Point is not a Capture Point, its margin of error is within the size of the foot. 


\subsection{Control Algorithms for the Stepping Stones Problem}

Now consider the problem of taking steps to desired locations. We refere to this problem as the One-Step Stepping Stones problem, since it is similar to crossing a pond over stones considering only one step at a time.

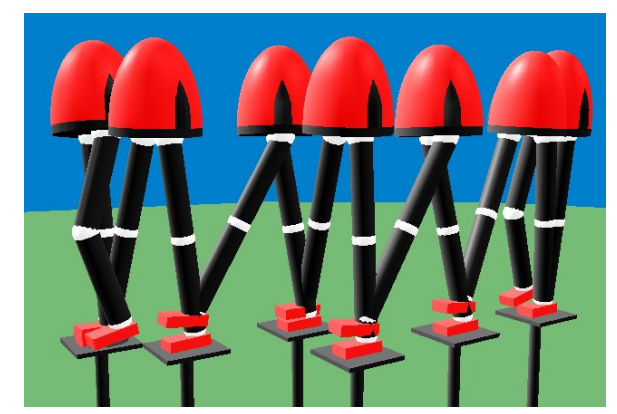

Fig. 4. Time elaspsed snapshots of simulated 12 degree-of-freedom lower-body biped walking over discrete steps while remaining One-Step Capturable. Snapshots are taken at one second intervals.

In a simulation study, we developed an algorithm for one-step stepping stones that uses the Center of Pressure on the support foot to guide the Capture Point out of the foot toward the desired stepping location when told to take a step. The swing leg then swings to the desired stepping location and is loaded once the estimated Capture Point becomes coincident with the desired step location. Figure 4 shows time elapsed snapshots of the simulated biped using this algorithm. Note that since the robot steps to a Capture Point, at all times it is One-Step Capturable. If the robot is not told to step to the next desired location, it will stop and balance on one leg.

Since the desired footholds are staggered, the motion is not periodic and hence Poincare return maps could not be used to demonstrate stability of this simulation. Since there is a toe-off phase, the ZMP criterion is violated on every step and is therefore not applicable to this simulation.

Even though the robot is One-Step Capturable throughout the simulation, the motion is dynamic, fairly natural-looking, moderate speed, and includes toe-off and double-support phases. If the robot is told the next step in advance, it does not need to stop over the current step and can continue at a moderate velocity. Therefore, being One-Step Capturable does not imply stopping or even imply slow walking. In this example the small performance degradation is well worth it in order to get the safety margin that being One-Step Capturable provides.

In current work we are investigating how speed can be increased by looking two or three steps ahead and guiding the capture point on a path that curves by the first stepping stone and then on to the second stepping stone as the first step is being taken. 


\section{Discussion and Future Work}

\subsection{Probabilistic Stability Margins}

In this paper, we have defined stability assuming a deterministic system. However, bipeds should be considered nondeterministic, since ground variations, sensor noise, and external disturbances are impossible to precisely model.

Most stability margins handle nondeterminism by relating to the tolerance to a particular unknown disturbance. This is the case for phase margins and gain margins in traditional linear control and is the case for many margins for bipedal walking, such as the static stability margin and the margins introduced in this paper.

These margins typically give comparative indications of robustness to terrain, noise, and disturbances (the larger the margin, the greater the disturbance that can be tolerated). They sometimes are an indication of the magnitude of the largest single disturbance that can be tolerated. However, they usually do not indicate the probability of instability given a particular disturbance distribution. In a companion paper [28], we explore stochastic stability margins for legged locomotion.

\subsection{Proofs of Conjectures}

In this paper we have stated several conjectures without proof. While these conjectures seem logical, it would be very useful to prove them since they are relied on for both the theory and the computational implementation in this paper. These conjectures are:

- In the limit as $\mathrm{N}$ approaches infinity, N-Step Capturable implies stability as defined by Definition 3.

- The Capture Region remains the same whether or not the constraint is enforced that the Center of Pressure remains at a Capture Point during a step.

- For flat terrain, the Capture Region will be a connected, and perhaps even convex, area on the ground.

- Once it has been determined to use reserve angular momentum to catch balance, immediately using all that is available as rapidly as possible is the strategy that results in the recovery from the worst conditions.

\subsection{Generalization}

In this paper we considered bipedal walking only. For a multi-legged robot or animal, similar definitions should apply. The difference would be that instead of requiring the foot to cover a Capture Point, the resultant convex hull of the support polygon would need to cover a Capture Point. For running, the difference would be in the Reachable Region of the swing foot. The Reachable Region would be larger, and in addition to being kinematically constrained, it would also be constrained by the leg strength and take-off velocities. 


\section{References}

1. Muhammad Abdallah and Ambarish Goswami. A biomechanically motivated two-phase strategy for biped upright balance control. IEEE International Conference on Robotics and Automation (ICRA), 2005.

2. Michael J. Coleman. A Stability-Study of a Three-Dimensional Passive-Dynamic Model of Human Gait. PhD thesis, Cornell University, 1998.

3. Steven H. Collins, Andy Ruina, Russ Tedrake, and Martijn Wisse. Efficient bipedal robots based on passive-dynamic walkers. Science, 307:1082-1085, February 182005.

4. Steven H. Collins, Martijn Wisse, and Andy Ruina. A three-dimensional passivedynamic walking robot with two legs and knees. International Journal of Robotics Research, 20(7):607-615, July 2001.

5. E.R. Dunn and R.D. Howe. Foot placement and velocity control in smooth bipedal walking. pages 578-583. IEEE International Conference on Robotics and Automation (ICRA), 1996.

6. Elena Garcia, Joaquin Estremera, and Pablo Gonzalez de santos. A classification of stability margins for walking robots. In Proceedings of the International Conference on Climbing and Walking Robots (CLAWAR), 2002.

7. A. Goswami. Postural stability of biped robots and the foot rotation indicator (FRI) point. International Journal of Robotics Research, 18(6), 1999.

8. A. Goswami, B. Espiau, and A. Keramane. Limit cycles and their stability in a passive bipedal gait. pages $246-251$. IEEE International Conference on Robotics and Automation (ICRA), 1996.

9. K. Hirai, M. Hirose, Y. Haikawa, and T. Takenaka. The development of Honda humanoid robot. In Proceedings of the IEEE International Conference on Robotics and Automation (ICRA), pages 1321-1326, 1998.

10. Qiang Huang, Kazuhito Yokoi, Shuuji Kajita, Kenji Kaneko, Hirohiko Arai, Noriho Koyachi, and Kazuou Tanie. Planning walking patterns for a biped robot. IEEE Transactions on Robotics and Automation, 17(3):280-289, June 2001.

11. S. Kajita and K. Tani. Study of dynamic biped locomotion on rugged terrainderivation and application of the linear inverted pendulum mode. volume 2, pages 1405 - 1411. IEEE International Conference on Robotics and Automation (ICRA), 1991.

12. Shuuji Kajita, Fumio Kanehiro, Kenji Kaneko, Kazuhito Yokoi, and Hirohisa Hirukawa. The 3d linear inverted pendulum mode: a simple modeling for a biped walking pattern generation. pages 239 - 246. IEEE International Conference on Intelligent Robots and Systems (IROS), 2001.

13. T. Kato, A. Takanishi, H. Jishikawa, and I. Kato. The realization of the quasidynamic walking by the biped walking machine. In A. Morecki, G. Bianchi, and K. Kedzior, editors, Fourth Symposium on Theory and Practice of Walking Robots, pages 341-351. Warsaw: Polish Scientific Publishers, 1983.

14. Hassan K. Khalil. Nonlinear Systems. Prentice Hall, 3rd edition, December 2001.

15. A.D. Kuo and F. E. Zajac. Human standing posture: multijoint movement strategies based on biomechanical constraints. Progress in Brain Research, 97:349-358, 1993.

16. Tad McGeer. Passive walking with knees. pages 1640 - 1645. IEEE International Conference on Robotics and Automation (ICRA), 1990. 
17. H. Miura and I. Shimoyama. Dynamic walk of a biped. International Journal of Robotics Research, 3(2):60-74, 1984.

18. Jun Morimoto and Christopher Atkeson. Minimax differential dynamic programming: An application to robust biped walking. Advances in Neural Information Processing Systems, 2002.

19. Hun ok Lim and Atsuo Takanishi. Waseda biped humanoid robots realizing human-like motion. pages 525-530. IEEE International Workshop on Advanced Motion Control, 2000.

20. Marko Popovic, Amy Englehart, and Hugh Herr. Angular momentum primitives for human walking: Biomechanics and control. In Proceedings of the IEEE/RSJ International Conference on Intelligent Robots and Systems, 2004.

21. Marko Popovic, Ambarish Goswami, and Hugh Herr. Ground reference points in legged locomotion: Definitions, biological trajectories and control implications. International Journal of Robotics Research, 24(12):1013-1032, Dec 2005.

22. Jerry Pratt. Exploiting Inherent Robustness and Natural Dynamics in the Control of Bipedal Walking Robots. PhD thesis, Computer Science Department, Massachusetts Institute of Technology, 2000.

23. Jerry Pratt and Gill Pratt. Intuitive control of a planar bipedal walking robot. In Proceedings of the IEEE International Conference on Robotics and Automation (ICRA), 1998.

24. Jerry Pratt, A. Torres, Peter Dilworth, and Gill Pratt. Virtual actuator control. IEEE International Conference on Intelligent Robots and Systems, 1996.

25. C.L. Shih and W.A. Gruver. Control of a biped robot in the double-support phase. IEEE Transactions on Systems, Man and Cybernetics, 22(4):729-735, 1992.

26. Michael Sipser. Introduction to the Theory of Computation. Course Technology, second edition, 2005.

27. Adam C. Smith and Matthew D. Berkemeier. Passive dynamic quadrupedal walking. In Proceedings of the 1997 IEEE Conference on Robotics and Automation, volume 1, pages 34-39. IEEE, April 1997.

28. Russ Tedrake and Jerry E. Pratt. Probabilistic stability in legged systems and the mean first passage time (FPT) stability margin. In progress, 2005.

29. Russ Tedrake, Teresa Weirui Zhang, Ming-fai Fong, and H. Sebastian Seung. Actuating a simple 3D passive dynamic walker. In Proceedings of the IEEE International Conference on Robotics and Automation (ICRA), volume 5, pages 4656-4661, New Orleans, LA, April 2004.

30. Miomir Vukobratovic and Branislav Borovac. Zero-moment point - thirty five years of its life. International Journal of Humanoid Robotics, 1(1):157-173, 2004.

31. E. R. Westervelt, J. W. Grizzle, and D. E. Koditschek. Zero dynamics of underactuated planar biped walkers. IFAC-2002, Barcelona, Spain, pages 1-6, Jul 2002.

32. Pierre-Brice Wieber. On the stability of walking systems. In Proceedings of the International Workshop on Humanoid and Human Friendly Robots, 2002.

33. Martijn Wisse. Essentials of dynamic walking; Analysis and design of two-legged robots. PhD thesis, Technische Universiteit Delft, 2004.

34. J. Yamaguchi, N. Kinoshita, A. Takanishi, and I. Kato. Development of a dynamic biped walking system for humanoid - development of a biped walking robot adapting to the humans' living floor. pages 232-239. IEEE International Conference on Robotics and Automation (ICRA), 1996. 
